\title{
Author reply - Letter to the Editor Re: Singh I et al: Growing skull fractures: guidelines for early diagnosis and surgical management
}

\author{
Ishwar Singh ${ }^{1}$
}

Received: 16 October 2016 / Accepted: 26 October 2016 / Published online: 26 November 2016

(C) Springer-Verlag Berlin Heidelberg 2016

Sir,

Thank you very much for your comments and subsequent discussion regarding the article. It is unanimously accepted that skull fracture with dural tear and entrapment of the arachnoid membrane or brain tissue within the fracture margin, is the most important factor for growing skull fracture pathogenesis [1]. The present study also corroborates this pathogenesis. Before this study, radiography and CT scanning were the most common methods used to assess and diagnose these cases. Unfortunately, they are not sufficient especially in early stages after trauma.

Prof. Paul Steinbok has raised the question that some patients with diastatic fracture with dural tear might heal and may not require surgery as he has observed in his practice. I slightly differ as he is not doing MRI, which has high predictive value for diagnosing dural tear which is essential for development of growing skull fracture. Moreover, if herniation of brain matter is there, which was seen in all operated cases in our study, the healing of fracture is unlikely. Diastatic fracture or brain contusion does not always cause dural tear/brain herniation which is essential for development of growing skull fracture as seen in four patients in our study. Another longterm longitudinal study in the future will answer whether diastatic fractures with dural tear heal spontaneously or always progress to growing skull fractures.

Secondly, a protocol was recommended in which only clinical signs and symptoms and skull radiography done at 4 weeks for diagnosing high-risk patients for growing skull fracture with reassessment after 2-3 months in equivocal cases. I believe that the time interval between head injury and diagnosis of growing skull fracture varies from 1 day to many years, and pulsatile mass or bony defect at the site of the known diastatic fracture is not always seen in early stages. Skull radiography can diagnose growing skull fracture if there is significant widening of fracture which is unlikely to be seen at 4 weeks in most cases. In my opinion, the MRI is essential for diagnosing growing skull fracture. I agree that getting an MRI is difficult in small infants and may require general anesthesia; but with early diagnosis, one can avoid complications like delayed neurological deficit, seizure, and cranioplasty.

\section{Compliance with ethical standards}

Conflict of interest There is no conflict of interest.

\section{References}

1. Singh I, Rohilla S, Siddiqui SA, Kumar P (2016) Growing skull fractures: guidelines for early diagnosis and surgical management. Child's nervous system: ChNS: official journal of the International Society for Pediatric Neurosurgery 32: $1117-1122$

Ishwar Singh

drishwarsingh@yahoo.co.in

1 PT.BD Sharma University of Health Sciences, Rohtak, Haryana, India 